\title{
Matérínia
}

Revista Matéria, v. 13, n. 4, pp. 617 - 623, 2008

ISSN 1517-7076

http://www.materia.coppe.ufrj.br/sarra/artigos/artigo10977

\section{Caracterização micrográfica de barras de ZTA infiltradas com vidro de lantânio e sua correlação com resistência à flexão por três pontos}

\author{
Pinto, V.B.B. ${ }^{\text {I}}$; Silva, P.T.G. ${ }^{\text {I }}$ Bica, M.V.R. ${ }^{\mathrm{I}}$, Nóbrega, M.C.S. ${ }^{\mathrm{I}}$, Ogasawara, T. ${ }^{\mathrm{I}}$ \\ I Laboratório de Cerâmicas Dentais - PEMM/COPPE/UFRJ CP: 68505 - 21941-972, Rio de Janeiro, RJ. \\ e-mail: bemfica@metalmat.ufrj.br, ptagasi@mealmat.ufrj.br, mnobr@metalmat.ufrj.br,
} marcusvrb@yahoo.com.br, ogasawat@metalmat.ufrj.br

\section{RESUMO}

Após a sua fratura em ensaio de flexão por três pontos, barras de In-Ceram ${ }^{\circledR}$ ZTA foram lixadas e polidas e , por microscopia eletrônica de varredura (MEV), foram avaliadas as quantidades relativas de fases que elas continham. Foram usadas 10 barras de $25,0 \mathrm{~mm}$ x $4 \mathrm{~mm}$ para cada uma das seguintes espessuras: 1,0 mm, 2,0 mm e 3,0 mm. Resultados: (a) Barras de In-Ceram ${ }^{\circledR}$ ZTA de $3 \mathrm{~mm}$ de espessura não conseguem alcançar uma completa infiltração de vidro de alumino-silicato de lantânio até o seu núcleo, nas condições operacionais recomendadas pelo fabricante (b) A MEV com elétrons retro-espalhados é de fato uma técnica adequada para o estudo da infiltração do vidro de lantânio em barras In-Ceram ${ }^{\circledR}$ ZTA.

Palavras-chaves: cerâmicas dentais, In-Ceram ${ }^{\circledR}$ ZTA, resistência à flexão, caracterização por MEV.

\section{Micrographic characterization of La-Glass Infiltrated ZTA bars and Its correlation to the three-point flexural stength}

\begin{abstract}
After their fracture at the end of three-point flexural strength test, Inceram ${ }^{\circledR}$ ZTA bars have been ground and polished and had their relative amounts of phases evaluated by scanning electron microscopy (SEM). Samples of 10 bars with $25.0 \mathrm{~mm}$ length and $4.0 \mathrm{~mm}$ width have been prepared for each of the following thickness: $1.0 \mathrm{~mm}, 2.0 \mathrm{~mm}$ and $3.0 \mathrm{~mm}$. Results: (a) $3 \mathrm{~mm}$ thick In-Ceram ${ }^{\circledR} \mathrm{ZTA}$ bars do not achieve a complete lanthanum alumino-silicate glass infiltration up to their core under operational conditions recommended by the manufacturer; (b) SEM with back-scattered electrons is effectively a suitable technique for studying infiltration of lanthanum glass into in-Ceram ${ }^{\circledR}$ ZTA bars.
\end{abstract}

Keywords: dental ceramics, In-Ceram ${ }^{\circledR}$ ZTA, flexural strength, SEM characterization.

\section{INTRODUÇÃO}

As cerâmicas odontológicas já constituem a opção mais estética para a substituição parcial ou total de tecidos dentários perdidos, sendo, no entanto, suscetíveis a fraturas quando expostas a tensões de tração ou flexão, devido à baixa tenacidade à fratura e limitada resistência mecânica quando comparada às ligas dentárias. Por isso, as próteses parciais fixas têm merecido intensivos estudos para minimizar os insucessos clínicos [1 - 5], ao mesmo tempo em que se obtém dureza similar à do esmalte dentário aliado à boa inércia química.

O método In-Ceram ${ }^{\circledR}$ consiste no pincelamento de barbotina (Slip Coating) do óxido de alumínio usado para construção da infra-estrutura, a qual é sinterizada parcialmente até obter uma microestrutura com poros abertos e interconectados. O material obtém sua resistência através da infiltração de vidro de lantânio no material poroso com um vidro de lantânio $[\underline{1}, \underline{6}, \underline{7}]$. Com a finalidade de aumentar a tenacidade à fratura do material $\left(\mathrm{K}_{\mathrm{Ic}}\right)$, óxido de zircônio pode ser adicionado [으 - $\left.\underline{10}\right]$, resultando em ZTA (alumina tenacificada pela zircônia). A adição de 33 \% de zircônia eleva a resistência à flexão até 630 ( \pm 58) MPa, enquanto que o $\mathrm{K}_{\mathrm{Ic}}$ dobra.

As ZTAs utilizadas em odontologia são indicadas para confecção de infra-estrutura de próteses parciais fixas posteriores (PPFP). O In-Ceram ${ }^{\circledR}$ Zircônia, ICZ, por ser um compósito de ZTA, possui propriedades mecânicas superiores àquelas do In-Ceram ${ }^{\circledR}$ Alumina. Essas propriedades superiores qualificam este material para ser utilizado em próteses parciais fixas posteriores [1, 11, 12]. 


\section{MATERIAIS E MÉTODOS}

Dez corpos de prova em formato de barras, de 25,0 mm de comprimento e de seção retangular de 4,0 mm de largura, foram preparados para cada uma das seguintes espessuras: 1,0 mm, 2,0 mm e 3,0 mm. Esta operação foi iniciada mediante pincelamento da barbotina do pó cerâmico Vita Inceram ${ }^{\circledR}$ Spinell sobre um molde de gesso de especial Vita, tendo dimensões 25,0mm (comprimento) x 4,00mm (largura horizontal) x 3,00mm (espessura vertical), inserido dentro de um molde de polivinilsiloxano Elite Double 8 (Zhermack, Itália), cuja cavidade tinha as seguintes dimensões: $25,0 \mathrm{~mm}$ de comprimento x 4,0mm de largura horizontal $\mathrm{x}$ $\mathrm{T} \mathrm{mm}$ de altura vertical. Os valores de $\mathrm{T}$ eram iguais a $4,0 \mathrm{~mm}, 5,0 \mathrm{~mm}$ e $6,0 \mathrm{~mm}$, respectivamente, para as espessuras de 1,0 $\mathrm{mm}, 2,0 \mathrm{~mm}$ e 3,0 $\mathrm{mm}$ das barras de corpos de prova a serem produzidos.

Alguns detalhes dos procedimentos acima referidos merecem ser destacados:

(a) Modelos de acrílico (de espessuras de 3, 4, 5 e $6 \mathrm{~mm}$, com 25,0 mm de comprimento e 4,0 mm de largura) foram usados na confecção dos moldes de polivinilsiloxano Elite Double 8 ( Zhermack, Itália);

(b) Esperou-se pelo menos 2 horas antes de retirar o modelo de gesso, já cristalizado, após o preenchimento do molde de polivinilsiloxano com o gesso Especial Vita;

(c) O modelo de gesso referido em (b) recebeu um acabamento com lamina de bisturi $\mathrm{n}^{\circ} 12$ e lixas $\mathrm{n}^{\circ} 600$ e 1200 (a seco), a fim de corrigir qualquer diferença entre este e o modelo de acrílico;

(d) Após o acabamento, o modelo de gesso especial foi posicionado dentro do molde de polivinilsiloxano (contendo cavidades de $25 \mathrm{~mm}$ x $4 \mathrm{~mm}$ x T mm), para confecção das barras precursoras de In-Ceram ${ }^{\circledR}$ Zirconia (ZTA);

(e) A barbotina da ZTA foi, a seguir, manipulada de acordo com as especificações do fabricante e transferida com pincel para dentro dos moldes de polivinilsiloxano, contendo no seu fundo uma barra de gesso para colagem;

(f) Durante o pincelamento da barbotina, o molde de polivinilsiloxano foi agitado por vibração, auxiliando um melhor escoamento da suspensão, de modo a reduzir a probabilidade de ocorrência de bolhas barra cerâmica resultante.

O conjunto modelo de gesso + barra cerâmica, resultante da colagem, foi posicionado em cima base de alumina do forno Inceramat $3 \circledR$ (VITA Zahnfabrik, Bad Säckingen, Alemanha), para a sinterização parcial, de acordo com a programação pré-estabelecida pelo fabricante.

Após a sinterização parcial, as barras de ZTA foram usinadas a seco com auxílio de pedras diamantadas a 5000 rpm, com o auxílio de micro-motor W\&H, MF-PERFECTA 9975, até alcançarem as dimensões corretas.

Após a usinagem, as barras cerâmicas foram posicionadas sobre uma lâmina de platina (95\% Pt + $5 \% \mathrm{Au}$ ), recobertas com a suspensão de vidro de alumino-silicato de lantânio em água bi-destilada e levadas ao forno Inceramat $3{ }^{\circledR}$ para queima destinada à infiltração de vidro. Em todas as barras, foi utilizada a mesma cor do vidro de lantânio (Z22), do lote nº 6491.

O excesso de vidro das amostras, ao final da queima de infiltração de vidro, foi removido com a utilização de pontas diamantadas (EVE-DIABOND - granulação grossa DB-15g) a 20000 rpm, com o auxílio do micro-motor W\&H, MF-PERFECTA 9975, seguindo sempre a mesma orientação de direção e sentido. Depois disso, aplicou-se nas barras cerâmicas jato de $\mathrm{Al}_{2} \mathrm{O}_{3}$ de $110 \mu \mathrm{m}$ de tamanho de partícula e pressão constante de 3,5 bar. Nesta operação, as amostras foram posicionadas, aproximadamente, a 2,5 a 3,0 cm de distância da saída do jato de alumina, que tinha uma inclinação de $45^{\circ}$ em relação à superfície a ser jateada

Após constatar, por meio de inspeção visual, que todo o excesso de vidro lantânio tinha sido removido, as barras cerâmicas foram posicionadas sobre uma lã de vidro e levadas ao interior do forno Vacumat $40 ®$, que já se encontrava pré-aquecido até $600^{\circ} \mathrm{C}$. Este forno foi, então, fechado e aquecido até $1000^{\circ} \mathrm{C}$ num tempo de 5 minutos, permanecendo no patamar final durante mais 5 minutos, antes do resfriamento. Eventuais vestígios de vidro remanescentes na superfície da barra cerâmica foram removidos, as arestas das barras foram levemente adoçadas e as superfícies foram polidas a seco, usando uma seqüência de lixas (de granulação de 220, 360, 600 e 1200) e o acabamento com pasta de diamante de granulação 2 a 4 $\mu \mathrm{m}$ (DIAMOND EXCEL, FGM).

A resistência à flexão uniaxial foi determinada por meio de teste de flexão por três pontos, de acordo com a norma ISO 6872 [13] , usando uma máquina de ensaios mecânicos (EMIC modelo DL 1000, São José dos Pinhais, Brasil), com velocidade de ensaio de $0,5 \mathrm{~mm} / \mathrm{s}$, estando as barras cerâmicas apoiadas sobre dois cilindros de aço distantes entre si de $14 \mathrm{~mm}$.. Estes apoios cilíndricos foram confeccionados com diâmetro de, aproximadamente, 1,5 vezes a espessura dos corpos de prova (ASTM, Designation C 1161-90) [14], conforme assinalados na Tabela 1.

A resistência à flexão por três pontos $(\mathrm{M})$ foi calculada usando a equação:

$$
\mathrm{M}=\left(3 \mathrm{Wl} /\left[2 \mathrm{bd}^{2}\right]\right)
$$


Onde W é carga de fratura (N); l é a distância entre os apoios do teste (mm); b e d são, respectivamente, a largura e espessura da barra cerâmica testada (mm).

Tabela 1: Diâmetro dos apoios cilíndricos de acordo com o grupo de teste

\begin{tabular}{c|c|c}
\hline Grupo & Espessura da barra cerâmica testada (mm) & $\begin{array}{c}\text { Diâmetro dos apoios cilíndricos } \\
(\mathrm{mm})\end{array}$ \\
\hline 1 & 1,00 & 1,4 \\
\hline 2 & 2,00 & 2,9 \\
\hline 3 & 3,00 & 4,5 \\
\hline
\end{tabular}

As barras cerâmicas, fraturadas no teste de flexão por três pontos, tiveram a sua seção de fratura lixada, polida e examinada por microscopia eletrônica de varredura (MEV). Micrografias representativas foram impressas em papel tamanho A3 e as quantidades relativas das fases presentes (poros, vidro infiltrado, grãos de zircônia e alumina) foram determinadas por corte (usando bisturi e tesoura), pesagem e cálculos. A pesagem de cada fração de recortes de papel foi feita numa balança analítica de quatro casas decimais. A integração da área em $\mathrm{l}_{\mathrm{i}}^{2} \mu \mathrm{m}^{2}$ (a escala micrométrica na micrografia permite calcular a área da micrografia em $\mu \mathrm{m}^{2} / \mathrm{g}$ ), onde $l_{i}$ é o lado de um cubo [de volume igual a $V_{i}=l_{i}^{3}$ ] representativo de uma particular fase representada na micrografia. $\mathrm{O}$ volume global $\mathrm{V}_{\mathrm{t}}$ de cerâmica representado na micrografia "tamanho A3" é igual à soma dos volumes parciais das fases. A porcentagem volumétrica de cada fase é \%Volumétrica $=\left(V_{i}\right.$ $\left./ \mathrm{V}_{\mathrm{t}}\right) \times 100$.

\section{RESULTADOS}

A Tabela 2 apresenta a média e o correspondente desvio padrão para cada um dos três grupos de barradas obtidos no teste de resistência à flexão uniaxial por três pontos.

Tabela 2: Resultados dos valores médios de força máxima, resistência à flexão e seu desvio padrão, no teste de flexão, por três pontos dos grupos analisados.

\begin{tabular}{c|c|c|c}
\hline & & Resistência à Flexão & \\
Grupo & Força máxima $(\mathrm{N})$ & 549,60 & Desvio Padrão (MPa) \\
\hline 1 & 104,69 & 424,77 & 87,34 \\
\hline 2 & 323,63 & 422,84 & 110,91 \\
\hline 3 & 714,66 & 78,04 \\
\hline
\end{tabular}

As superfícies de fratura dos corpos de prova foram analisadas por meio de microscopia eletrônica de varredura (MEV - JOEL 6460LV), com a utilização de elétrons retro-espalhados, associados com mapeamento dos elementos presentes em cada grão por meio de EDS. Isto permitiu identificar inequivocamente cada um dos grãos presentes em cada micrografia MEV considerada para avaliação quantitativa das fases alumina, zircônia, vidro de lantânio e poros (vide ilustração na Figura 1).

A Tabela 3 mostra as porcentagens volumétricas das fases associadas às micrografias de elétrons retro-espalhados: a porcentagem de vidro infiltrado decresce com o aumento da espessura da barra cerâmica.

Tabela 3: Porcentagens volumétricas de fases associadas às micrografias dos grupos 1, 2 e 3 de InCeram ${ }^{\circledR}$ ZTA.

\begin{tabular}{l|l|l|l|l}
\hline \multirow{2}{*}{ Barras cerâmicas } & \multicolumn{4}{|c}{ \% Volumétricas de fases } \\
\cline { 2 - 5 } & Poros & Vidro & Alumina & Zircônia \\
\hline Grupo(1): 1-1 & 0,51 & 15,19 & 62,70 & 21,60 \\
\hline Grupo(2): $2-1$ & 0,09 & 5,25 & 80,82 & 13,83 \\
\hline Grupo(3): 3-1 & 0,50 & 6,61 & 74,62 & 18,27 \\
\hline Grupo(3):3-2 borda & 0,14 & 11,42 & 67,44 & 20,99 \\
\hline
\end{tabular}



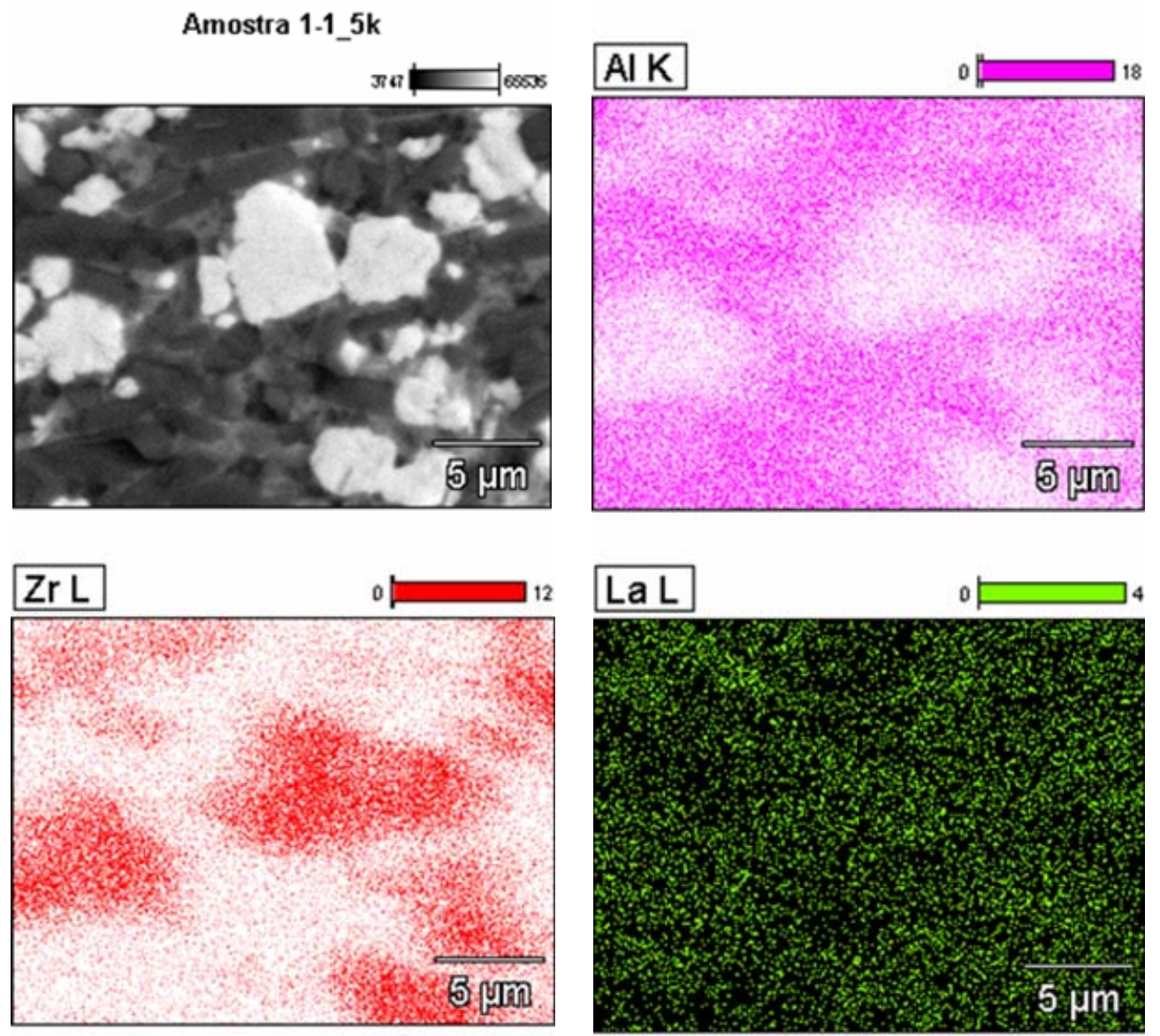

Figura 1: Análise de EDS - mapeamento dos elementos [Al representa alumina ( $\mathrm{x}=2, \mathrm{y}=2), \mathrm{Zr}$ representa zircônia tetragonal $(x=1, y=1)$ e La representa o vidro $(x=2, y=1)]$ referentes nos grãos da micrografia MEV $(\mathrm{x}=1, \mathrm{y}=2) ; \mathrm{x}=$ abcissa dos quadrantes da Figura 1; $\mathrm{y}=$ ordenada dos quadrantes da Figura 1.

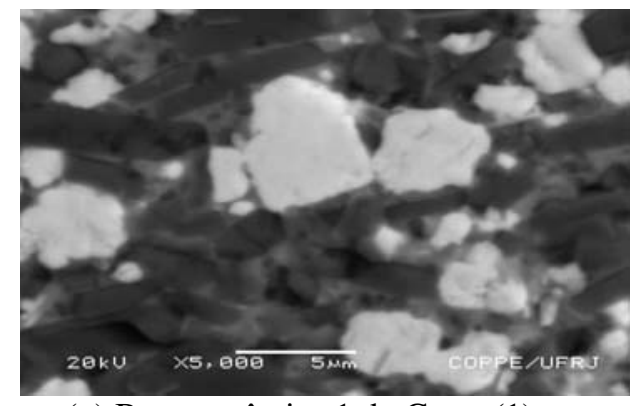

(a) Barra cerâmica 1 do Grupo(1)

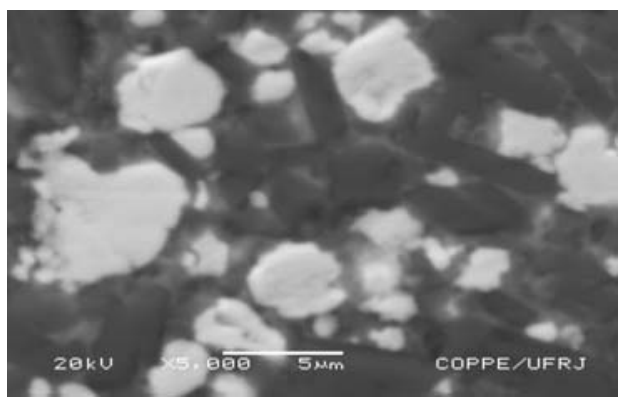

(b) Barra cerâmica 1 do Grupo(2)

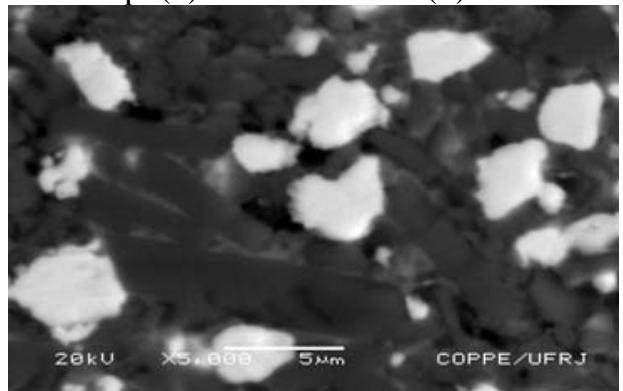

(c) Barra cerâmica 1 do Grupo(3)

Figura 2: Micrografias representativas de barras cerâmicas dos Grupos 1, 2 e 3 (elétrons retroespalhados): Fase branca = zircônia, cinza escura $=$ alumina, preta $=$ poro vazio e cinza claro = vidro de alumino-silicato de lantânio (conforme identificação prévia por EDS). 
A Figura 2 mostra micrografias representativas de barras cerâmicas dos Grupos 1, 2 e 3, tendo como fases contidas: poros (fase preta), alumina (fase cinza escura), vidro (fase cinza clara) e zircônia tetragonal (fase branca). O recorte da impressão micrográfica A3 correspondente a uma destas fases foi pesada separadamente.

Cabe assinalar que, por exemplo, a partir da escala micrométrica de $5 \mu \mathrm{m}$ correspondendo a 4,4 cm, numa micrografia tamanho A3 medindo (excluídas as bordas) 29,7 cm x 42,1 cm e pesando 9,7230g, ter-se-á $1,13636 \mu \mathrm{m}$ por centímetro de micrografia, correspondendo a 1,2963 $\mu \mathrm{m} 2$ por $\mathrm{cm} 2$ de micrografia. Mais ainda, ter-se-á 128,2 cm2/g de micrografia impressa em tamanho A3, correspondendo a 165,5475 $\mu \mathrm{m} 2 / \mathrm{g}$ de micrografia impressa em tamanho A3. Esta razão de $\mu \mathrm{m} 2 / \mathrm{g}$ se aplicada cada uma das fases presentes. Supondo uma distribuição homogênea das fases nas três dimensões, o cubo da raiz quadrada de cada área parcial (correspondente a uma particular fase) dará o volume da mesma fase considerada, representada na particular micrografia. As soma dos volumes parciais das fases dá o volume total de material da barra representado na referida micrografia. Daí, calcula-se a porcentagem de cada fase, dada na Tabela 3.

\section{DISCUSSÃO}

Inaugurou-se aqui uma nova metodologia que, apesar de extremamente trabalhosa (muitas horas de análise direta não instrumental de uma considerada micrografia em tamanho A3), constitui notável melhoria em relação à comparação a olho nu de micrografias justapostas. Naturalmente, o método deve ser repetido várias vezes numa mesma amostra (por exemplo, a coleta de 10 micrografias em regiões diferentes de cada corpo de prova [barra cerâmica, no presente caso]), a fim de avaliar a dispersão da micrografia, bem como utilizar várias amostras físicas (por exemplo: 10 corpos de prova de um mesmo lote) de maneira a viabilizar a avaliação da homogeneidade (ou a dispersão) dentro de um único lote. Neste assinalado quadro, seriam 100 micrografias em tamanho A3 para um lote de 10 amostras físicas, cada análise ceramográfica quantitativa levando muitas horas de trabalho do analista. Como anunciado acima, é realmente um trabalho que demanda muito tempo e paciência e, no futuro, enseja a sua substituição (total ou parcial) por uma análise ceramográfica quantitativa instrumentada (ou informatizada).

Conforme detalhadamente analisado em sua pesquisa de dissertação de Mestrado, Ruybal Bica [15] mostrou que o teste de Tukey revelou que o resultado do grupo 1 diferiu-se estatisticamente dos demais grupos, enquanto não houve diferença significativa entre os grupos 2 e 3. Resultados semelhantes foram encontrados por KELLY [16], que observou valores maiores de resistência à flexão uniaxial por três pontos em corpos de prova de menor espessura, quando comparadas amostras de 0,5 a 3,0 mm [16]. Foram obtidos, no presente estudo, valores de resistência à flexão que variaram de 549,60 ( \pm 87,34) MPa, 424,77 $( \pm 110,91)$ MPa e 422,84 ( \pm 78,04) MPa, respectivamente para os grupos 1, 2 e 3.

Valores de até 630 ( \pm 58$) \mathrm{MPa}$, foram descritos nos trabalhos de GUAZZATO et al [므, $\underline{9}$ ], com a utilização de amostras tendo dimensões de 1,2 x 4 × $20 \mathrm{~mm}$ (espessura x largura x comprimento). Estes valores, claramente, são superiores aos $549,60 \mathrm{MPa}$ do presente trabalho. Mas, em outro trabalho GUAZZATO et al [19] que estudou a influência dos tratamentos de superfície e térmico em corpos de prova In-Ceram Zirconia (ICZ) com dimensões de 1,2 x 4 x $20 \mathrm{~mm}$ (espessura x largura x comprimento), os resultados de resistência à flexão variaram de 452 ( \pm 35$)$ MPa para o grupo em que foi realizado apenas o jateamento da superfície até $563( \pm 62)$ MPa para as amostras que foram usinadas paralelamente ao longo eixo e depois sofreram tratamento térmico. Estes valores são muito parecidos com os do presente trabalho para uma equivalente espessura de barra cerâmica.

Já ANUSAVICE [1] descreve o valor resistência à flexão uniaxial por três pontos para o ICZ como sendo igual a $700 \mathrm{MPa}$. Isto significa que resultados melhores podem ser obtidos num trabalho de processamento cerâmico e de acabamento bem mais refinado do que empregado no presente estudo.

Os módulos de Weibull no presente estudo variaram de acordo com o grupo, sendo que o grupo 1 apresentou resultado de $m=7$, enquanto os resultados dos grupos 2 e 3 foram respectivamente $m=3,80$ e $\mathrm{m}=4$,73. O módulo de Weibull ( $\mathrm{m}$ ) é a distribuição de resultados. Este pode ser calculado por meio do coeficiente angular (inclinação) da reta de probabilidade acumulada. Quanto maior for o valor de m, menor a dispersão de resultados e consequentemente maior confiabilidade do material [풀, $\underline{18}]$.

LÜTHY et al [17] realizaram um estudo sobre resistência mecânica e confiabilidade de próteses parciais fixas posteriores totalmente cerâmicas. Os materiais utilizados para confecção das amostras foram: Cercon blanks (CEZ) (DeguDent, Hanau, Germany) t- $\mathrm{ZrO}_{2}$ (3\% mol - Y2O3), In-Ceram blanks (ICZ) (Vita Zahnfabrik, Bad Säckingen, Germany) $\mathrm{t}-\mathrm{ZrO}_{2}(12 \%$ mol - CeO2) e IPS Empress2 (E2) (Ivoclar, Schaan, Liechtenstein) dissilicato de lítio. Os autores concluíram que o CEZ possui capacidade média de carregamento de $706 \mathrm{~N}$ que é 1,5 vezes maior do que a do ICZ (407 N) e 2,7 vezes maior que a do E2 (260 $\mathrm{N})$. Os resultados do módulo de Weibull demonstraram que o CEZ apresenta menor dispersão dos resultados $(m=7)$, ou seja, maior confiabilidade em relação ao E2 $(m=5,7)$ e ICZ $(m=4,5)$. Portanto, os resultados do presente trabalho são comparáveis aos destes outros autores. 
De acordo com as patentes americanas [20, 21] de TYSZBLAT e com os dados do fabricante, os óxidos que podem estar presentes nos pós são: $\mathrm{Al}_{2} \mathrm{O}_{3}, \mathrm{SiO}_{2}, \mathrm{~B}_{2} \mathrm{O}_{3}, \mathrm{La}_{2} \mathrm{O}_{3}, \mathrm{CaO}, \mathrm{ZrO}_{2}, \mathrm{TiO}_{2}, \mathrm{CeO}_{2}$ e $\mathrm{Y}_{2} \mathrm{O}_{3}$. Nas análises por EDS, realizadas neste trabalho, da ZTA parcialmente sinterizada e infiltrada com vidro verificou-se a presença dos elementos químicos: Zircônio (Zr), Alumínio (Al), Oxigênio (O), Silício (Si), Cálcio (Ca), Sódio (Na) e Lantânio (La). Na técnica de EDS, apenas elementos químicos com massa atômica superior ao do Boro e que estejam presentes em concentrações superiores a 1\% são detectados. O provável é que a natureza particular do vidro empregado deverá acarretar diferenças no resultado final de resistência à flexão, por isso um estudo mais focado neste aspecto merece ser realizado.

$\mathrm{O}$ que se espera que aconteça com o aumento da espessura da amostra, mantendo-se constante o tempo e a temperatura de infiltração é que a profundidade de penetração do vidro seja a mesma em todas as amostras e, portanto, as amostras mais espessas terão menor fração da seção reta infiltrada pelo vidro de lantânio, enquanto o cálculo da resistência à flexão é feito considerando toda a seção reta da amostra. Daí, espessuras maiores resultam com menores resistências à flexão. Naturalmente, espessuras maiores requerem tempos maiores de infiltração e o uso do vácuo desde o início do aquecimento (para deslocar o ar inserido nos poros); um pequeno aumento de temperatura $\left(10\right.$ a $\left.15^{\circ} \mathrm{C}\right)$ também deveria ajudar.

Naturalmente a resistência à flexão em amostras In-Ceram ${ }^{\circledR}$ contém duas contribuições: (a) a da cerâmica parcialmente sinterizada e porosa; e (b) a do vidro de alumino-silicato de lantânio sob compressão do arcabouço cerâmico.

Em espessura muito grande a proporção de seção reta onde existe vidro infiltrado decresce proporcionalmente à espessura. Por isso, a partir de certa espessura de amostra a resistência à flexão da mesma é ditada predominantemente pelo arcabouço cerâmico poroso tornando-se desprezível a contribuição da fase vítrea. Esta tendência explica, até certo ponto, a inexistência de diferença significativa entre os valores de resistência à flexão dos grupos 2 e 3.

\section{CONCLUSÕES}

(a) Constatou-se que a espessura do corpo de prova de ZTA infiltrado com vidro de lantânio influenciou no valor de resistência à flexão uniaxial por três pontos porque a quantidade de vidro infiltrada diminui com a espessura do corpo de prova.

(b) A MEV com elétrons retro-espalhados é de fato uma técnica adequada para o estudo da infiltração do vidro de lantânio em barras In-Ceram ${ }^{\circledR}$ ZTA.

\section{AGRADECIMENTOS}

Os autores agradecem ao CNPq, CAPES, FAPERJ e Pronex CNPq-FAPERJ de Cerâmicas Dentais (Proc. E-26/171.204/2003) e a todos que direta ou indiretamente contribuíram para o sucesso desta pesquisa.

\section{BIBLIOGRAFIA}

[1] ANUSAVICE, K.J., Materiais Dentários, 11 ed., Rio de Janeiro, Elsevier Editora Ltda, 2005.

[2] CAMPBELL, S.D., SOZIO, R.B., "Evaluation of the fit and strength of an all-ceramic fixed partial denture”, Journal of Prosthetic Dentistry, v. 59, n. 3, pp. 301-306, 1998.

[3] KAMPOSIORA, P., et al., "Stress concentration in all-ceramic posterior fixed partial denture", Quintessence International, v. 27, n. 10, pp. 701-706, 1996.

[4] Magne, P., et al. "Stress distribution of inlay-anchored adhesive fixed partial dentures: A finite element analysis of the influence of restorative materials and abutment preparation design”, Journal of Prosthetic Dentistry, v. 87, pp. 516-527, 2002.

[5] OH, W., Anusavice, K.J., "Effect of connector design on the fracture resistance of all-ceramic fixed partial dentures”, Journal of Prosthetic Dentistry, v. 87, pp. 536-542, 2002.

[6] BOTTINO, M.A., et al, Estética em reabilitação oral metal free, 1 ed, São Paulo, Artes Médicas na Estante Virtual, 2000.

[7] CRAIG, R.G., POWERS, J.M., Materiais Dentários Restauradores, 11 ed., São Paulo, Livraria e Editora Santos, 2004. 
[8] GGUAZZATO, M., et al., "Strength, fracture toughness and microstructure of a selection of all-ceramic materials. Part II. Zirconia - based ceramics”, Dental Materials, v. 20, pp. 449-456, 2004.

[9] GUAZZATO, M., et al., "Strength, reliability and mode of fracture of bilayred porcelain/zircônia (YTZP) dental ceramics”, Biomaterials, v. 25, n. 20, pp. 5045-52, 2004.

[10] SZUTKOWSKA, M., "Fracture resistance behavior of alumina-zirconia composites", Journal of Materials Processing Technology, v. 153-154, pp. 868-874, November 2004.

[11] CASELLAS, D., et al., "Fracture toughness of zirconia-alumina composites", International Journal of Refractory Metals \& Hard Materials, v. 17, n. 1-3, pp. 11-20, 1999.

[12] DE AZA, A.H., et al., "Crack growth resistance of alumina, zirconia and zirconia toughened alumina ceramics for joint prostheses”, Biomaterials, v. 23, n. 3, pp. 937-945, 2002.

[13] ISO 6872:1995(E). Dental Ceramic International Standard

[14] American Society for Testing and Materials. ASTM C1161-02C. Standard Test Method for Flexural Strength of Advanced Ceramics at Ambient Temperature. Philadelphia: American Society for Testing and Materials, 2002.

[15] RUYBAL BICA, M.V., "Influência da espessura do corpo de prova sobre o valor da resistência à flexão por três pontos de um ZTA infiltrado com vidro de lantânio”, Dissertação de M.Sc. em Eng. Metalúrgica e de Materiais, COPPE/UIFRJ, Rio de Janeiro, RJ, Brasil, 2006.

[16] KELLY, J.R., "Clinically relevant approach to failure testing of all-ceramic restorations", Journal of Prosthetic Dentistry, v. 81, n. 6, pp. 652-661, 1999.

[17] LÜTHY, H., et al., "Strength and reliability of four-unit all-ceramic posterior bridges", Dental Materials, v. 21, n. 10, pp. 930-937, 2005.

[18] SULLIVAN, J.D., LAUZON, P.H., "Experimental probability estimators for weibull plots”, Journal of Materials Science Letters, v. 5, n.12, pp. 1245-1247, 1986.

[19] GUAZZATO, M., et al., "Influence of surface and heat treatments on the flexural strength of a glassinfiltrated alumina/zirconia-reinforced dental ceramic”, Dental Materials, v. 21, n. 5, pp. 454-463, 2005.

[20] TYSZBLAT, M., "Process for the preparation of a dental prosthesis by slight solid phase fritting of a metal oxide based infrastructure”, United States Patent n. 4, 772, 436, USA, September 1988.

[21] TYSZBLAT, M., "Process for the preparation of a dental prosthesis and the prosthesis produced by said process”, United States Patent, n. 5, 250, 352, USA, October 1993. 